Lepr Rev (1992) 62, 317-318

\title{
Editorial
}

\section{REVISED ESTIMATES OF GLOBAL LEPROSY NUMBERS}

The introduction and promotion of multidrug therapy (MDT) in the early $1980 \mathrm{~s}^{1}$ gave new life to efforts to control leprosy. ${ }^{2}$ Two more recent events have generated increased optimism with respect to that objective. The first was setting of the goal by the World Health Assembly to eliminate leprosy as a public health problem by the year 2000, defined as reducing the prevalence of the disease to less than one case per 10,000 population. The second has been the dramatic downwards revision of the estimated number of leprosy cases in the world, from the figure of 10-12 million that was used in the mid-1980s to a new figure of 5.5 million. $^{3}$

At first sight, this seems to be rapid progress indeed towards the elimination goal, but considerable caution must be exercised in interpreting the apparent fall in cases of the disease. In revising the figures the definition of an individual with leprosy has also been changed and this is responsible, in considerable part, for the reduced number of cases reported. The new figure of 5.5 million is the estimated number of patients still in need of chemotherapy. In addition, it is estimated that there are another 2-3 million individuals who no longer require chemotherapy but who have been left with residual deformities and who may require medical treatment and care. These individuals were not considered separately in the previous global estimate of leprosy burden and, indeed, many of them would still have been receiving dapsone monotherapy. Even so, the revised figure for the number of persons with active disease means that there has been a substantial reduction in the estimated prevalence of leprosy. It should be emphasized, however, that the decline in prevalence does not necessarily correspond to a fall in the incidence of the disease.

In situations where the rates of a disease are not changing very much over time there is a simple epidemiological relationship between prevalence (the number of cases of disease at a given point in time) and incidence (the number of new cases of disease arising in a given time period), which is that: prevalence $=$ incidence $\times$ average duration of disease. Thus a fall in prevalence may be brought about either by reducing the number of new cases or by reducing the length of time individuals have the disease. The major change in the WHO estimates of leprosy prevalence is due to the latter effect. Because a large proportion of leprosy patients are cured within 1 or 2 years with multidrug therapy, they cease to be classified as prevalent cases after that time, whereas with monotherapy they may have been classified as a case for many years. It has been possible, therefore, to remove many old cases from leprosy registers.

The 'elimination' goal requires that there must be a marked fall in the incidence of leprosy. It is hoped that high coverage and prompt treatment of prevalent and new cases 
of leprosy with multidrug therapy will rapidly render them non-infectious to others and thus prevent the secondary cases they would otherwise have caused. While this seems a reasonable expectation, the direct evidence that such therapy reduces the incidence of leprosy in a population is scanty and more time will have to pass before a definitive assessment can be made of the impact of multidrug therapy on transmission. There have been undoubted falls in the incidence of leprosy in some, though not all, parts of the world, but chemotherapy is certainly not the only possible explanation for the declines. ${ }^{4}$ Many of the falls started before multidrug therapy was widely available. Changing socioeconomic circumstances were major determinants of leprosy declines in the now developed countries ${ }^{5}$ and this is likely to be a component in the declines in incidence in some developing countries. It is also likely that the great expansion in the coverage of BCG vaccination in most developing countries has had important effects in those situations in which it appears to offer substantial protection against leprosy, such as in Africa $^{6,7}$ and Latin America. ${ }^{8,9}$

The optimism that the 'end' of leprosy is in sight has both good and bad aspects. It has encouraged a new enthusiasm for widespread employment of effective chemotherapy and strenuous efforts are being made to expand the application of multidrug therapy. Between 1986 and 1990 the number of patients on multidrug therapy increased from under half a million to over 2 million. ${ }^{2}$ A more negative aspect is the possible diminution of the importance attached to leprosy as a long-term health problem. A consequence of this may be that both private and public support for research on leprosy may fall. This will affect particularly those types of research which are unlikely to bear fruit until after the year 2000 , such as work on new vaccines. If leprosy does indeed cease to be a public health problem by that year, reduced support for long-term research may well seem wise in retrospect. But not all are convinced that the WHO target will be achieved. It now seems clear that the much more ambitious WHO target of health for all by that year is likely to have a very hollow ring. The more specific target for leprosy may be achievable but it is too soon to loose sight of the fact that a longer term perspective to control may be required.

Department of Epidemiology and Population Sciences

P G SMITH

London School of Hygiene and Tropical Medicine

Keppel Street, London WC1E $7 H T$

\section{References}

1 WHO Technical Report Series, No. 675, 1982 (Chemotherapy of leprosy for control programmes: report of a WHO Study Group).

2 Noordeen SK. Leprosy control through multidrug therapy (MDT). Bull WHO, 1991; 69: 263-9.

3 Noordeen SK, Lopez Bravo L, Sundaresan TK. Estimated number of leprosy cases in the world. Bull WHO, 1992; 70: (i) 7-10.

4 Fine PEM. Reflection on the elimination of leprosy. Int J Lepr, 1992; 60: 71-80.

5 Fine PEM. Leprosy - the epidemiology of a slow bacterium. Epidemiol Rev, 1982; 4: 161-88.

6 Stanley SJ, Howland C, Stone MM, Sutherland I. BCG vaccination of children against leprosy in Uganda: final results. $J$ Hyg (Camb), 1981; 87: 223-48.

7 Ponnighaus JM, Fine PEM, Sterne JAC, et al. Efficacy of BCG vaccine against leprosy and tuberculosis in northern Malawi. Lancet, 1992; 339: 636-9.

8 Convit J, Sampson C, Zuniga M, et al. Immunoprophylactic trial with combined $M$ ycobacterium leprae/BCG vaccine against leprosy: preliminary results. Lancet, 1992; 339: 446-50.

9 Rodrigues MLO, Silva SA, Neto JCA, et al. Protective effect of intradermal BCG against leprosy. A casecontrol study in central Brazil. Int J Lepr, 1992; 60: in press. 